\title{
Retroperitoneal Hamartoma: A Rare Entity
}

\author{
Sigdel GS', Karki K², Koirala U², Joshi BD², Dhital SP² \\ 'Department of Urology, College of Medical Sciences Teaching Hospital, Chitwan, Nepal. ${ }^{2}$ Department of General Surgery, \\ Kathmandu Model Hhospital, Kathmandu, Nepal
}

\section{ABSTRACT}

Retroperitoneal hamartoma in adult is an extremely rare entity. Here we report a case of 23-years-old lady who presented with alarge retroperitoneal mass and abdominal pain. The mass was surgically removed and the diagnosis of hamartoma was made on histological findings.

\section{Keywords: hamartoma malformative tumor, retroperitoneal}

\section{INTRODUCTION}

Hamartoma Refers to an excessive but focal overgrowth of cells and tissues native to the organ in which it occurs. ${ }^{1}$ Harmartoma can be thought of as the linkage between malformations and benign neoplasms, though the demarcation between the two at times is difficult and variously interpreted. Hamartoma commonly affects children and can develop in different parts of the body. Retroperitoneal hamartoma in an adult is a rarely reported entity in the literature.

\section{CASE REPORT}

A 23-year-old lady presented with a localized, dull aching, intermittent pain in the left flank for two years. Patient had no bowel and bladder symptoms. There was no loss of weight or appetite. Abdominal examination revealed aproximately $6 \times 6 \mathrm{~cm}$, non-pulsatile, fixed, retroperitoneal mass with smooth and regular margins in the left lumbar region. The complete blood count, biochemical investigation and other laboratory parameters were within normal limits. CT scan of abdomen disclosed a well-defined,hypodense mass in left lumbar region anterior and medial to the left kidney.
The mass was completely resected through the transperitoneal approach. It was a $5 \times 6 \mathrm{~cm}$ smooth, well encapsulated mass, situated just medial to the left kidney. The mass was supplied with multiple dilated vessels from its medial side (Figure1).

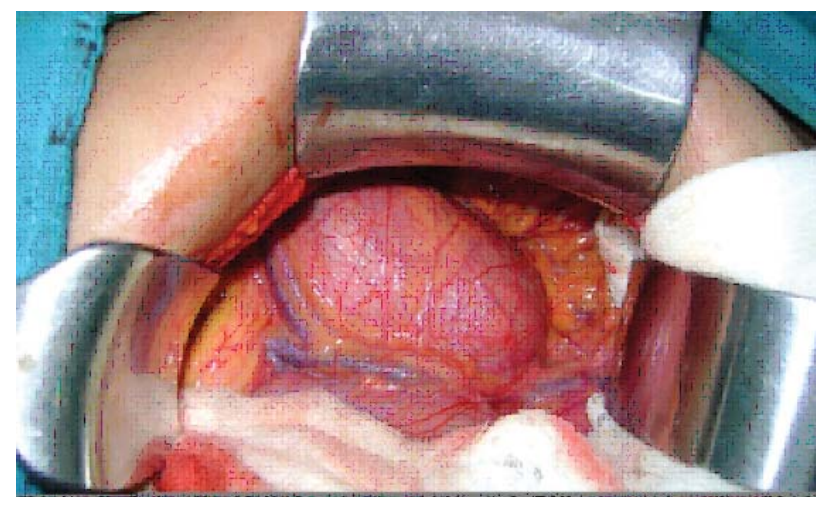

Figure 1. Retroperitoneal mass with dilated blood vessels

It was free from surrounding solid organs and great vessels.

Correspondence:

Dr. Ghanashyam Sigdel

Department of Urology, College of Medical Sciences Teaching Hospital, Chitwan, Nepal

Post box no: 23

E-mai: sigdelgs@yahoo.com 


\section{Histopathological findings}

Grossly, the mass was well encapsulated with smooth external surface. Cut surface showed multiple septations with prominent blood vessels and blood clots inside the mass (Figure 2).

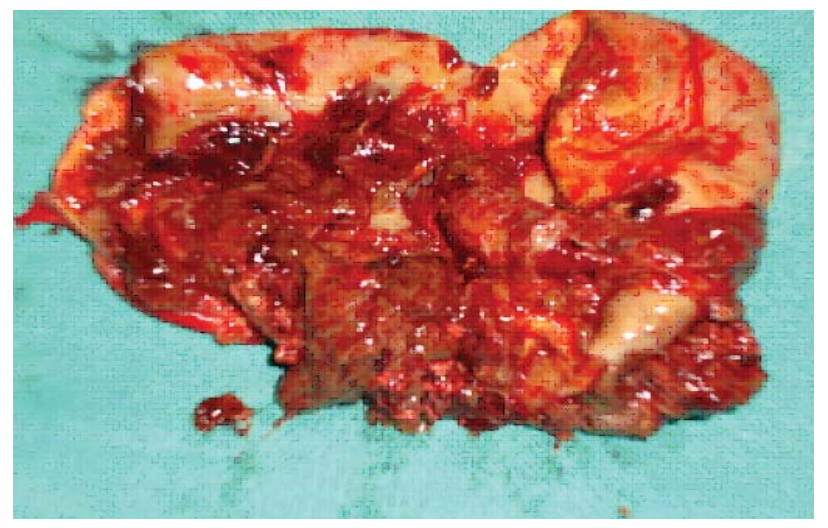

Figure 2. Cut section of the mass showing multipleseptations with blood vessels and clots inside

Microscopic examination showed disorganized pattern of tissue consisting of smooth muscles, fibrous tissues, adipose tissues and blood vessels. (Figure3).
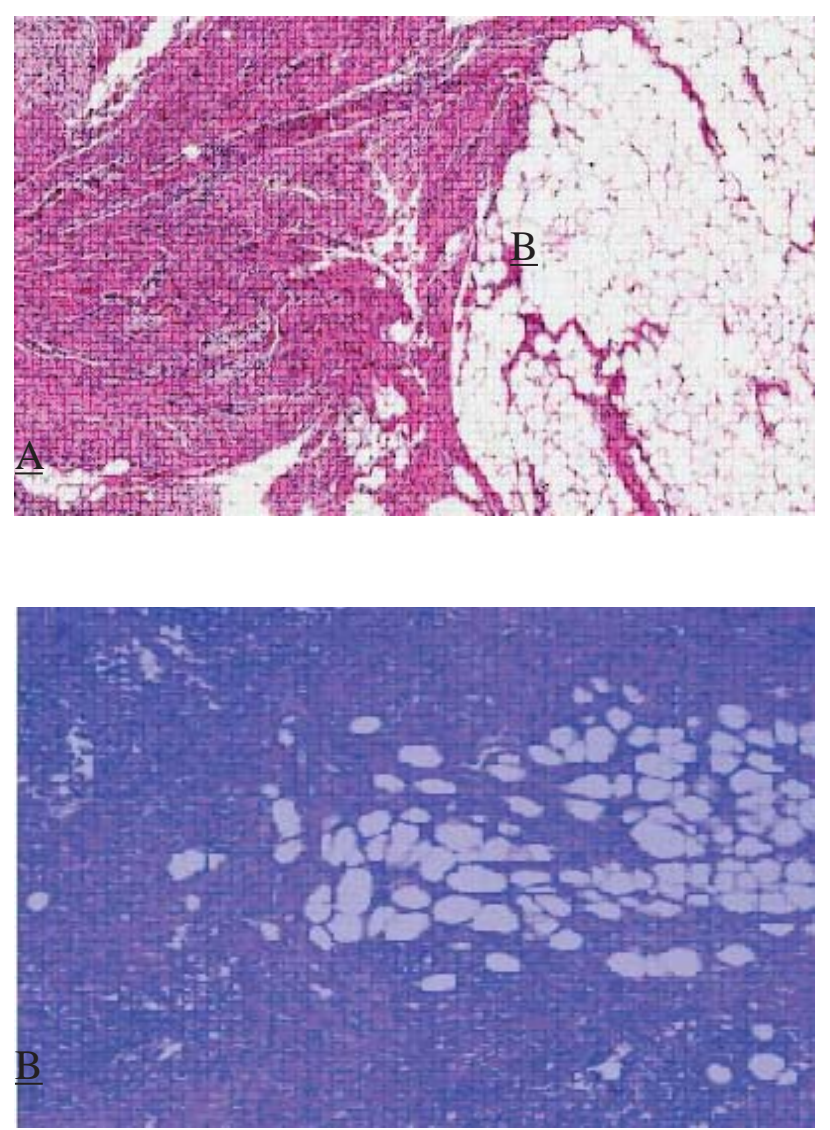

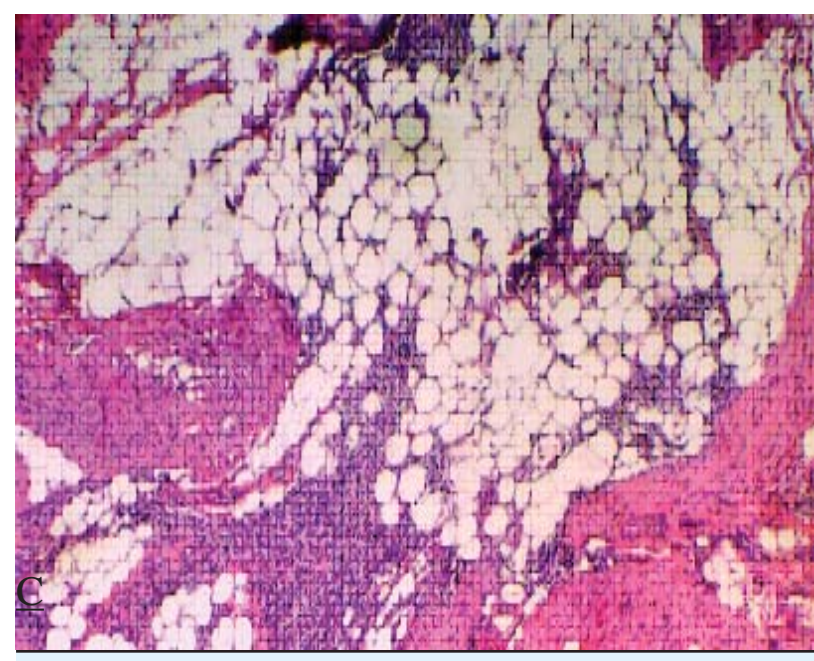

Figure 3. Micrograph showing all the elements of a hamartoma- smooth muscle, fibrous tissue, adipose tissue and blood vessels with a disorganized growth pattern (HE stain, $\mathrm{X} 400$ )

The case was diagnosed as retroperitoneal hamartoma.

\section{DISCUSSION}

Aberrant differentiation of cells may produce a mass of disorganized but mature specialized cells or tissue indigenous to the particular sites, referred to as a hamartoma. ${ }^{1}$ Although the cellular elements are mature and identical to those found in the remainder of the organ, they do not reproduce the normal architecture of the surrounding tissue. Due to their increased frequency of occurrence in infancy and childhood, many of them are believed to be developmental aberrations. Hamartoma may develop as an isolated pathology or as a component of some syndrome. Retroperitoneal hamartoma is an exceptional, benign, malformative tumor. ${ }^{2}$ They are usually asymptomatic, but may present as intermittent partial intestinal obstruction, peripheral edema and, poorly localized abdominal pain. ${ }^{3}$ Pathologically most of the retroperitoneal hamartoma are angiomatous lymphoid hamartoma ${ }^{4-6}$ CT scan is the most sensitive imaging modality for detecting these tumors. Cystic hamartoma usually are small, round, sharply marginated, and of homogeneous soft tissue density. ${ }^{7}$ It may appear as a heterogeneously enhancing mass with internal vessels and different level of attenuation according to the type of tissues present. ${ }^{8}$ Genetic analysis may reveal mutations in different genes that are linked to hamartoma and other associated malformations. Phosphatase and tensin homologue (PTEN) is a tumor suppressor gene recently implicated in a collection of disorders termed the PTEN hamartoma-tumor syndrome.9,10 Treatment of choice 
is complete surgical removal of the lesion, that leads to recovery without any sequel. ${ }^{2}$ Histological diagnosis of hamartoma is made on the basis of presence of fat, blood vessels, smooth muscle, striated muscle, cartilage, lymphoid and hematopoietic tissue in the tumor. ${ }^{1}$ We have reported Rretroperitoneal hamartoma in adult, which is a rare entity. The patient presented with vague abdominal symptoms and total excision of the mass was curative.

\section{REFERENCE}

1. Kumar,Abbas,Fausto:Robbins and CotranPathologic basis of disease.7thEdition. Saunders, Philadelphia,2004.P 498.

2. Martelli H, Revillon Y, Lortat-Jacob S, Pellerin D Retroperitoneal hamartoma.World J Surg.1985;9,2:310-5.

3. Richard Hellstrom R, Edwin R. Fisher. Benign retroperitoneal hamartoma .AMA Arch Surg. 1959;78,6:975-78.

4. Hamilton S, McInerney D. Retroperitoneal angiomatous lymphoid hamartoma demonstrated by ultrasound.Br J Radiol. 1981;54,645:813-5.

5. Gupta S, Kumar A, Khanna S .Retroperitoneal angiomatoushamartoma.Ann ChirGynaecol. 1980; 69,4:154-6.

6. Mallory A, Spink WW.Angiomatous lymphoid hamartoma in the retroperitoneum presenting with neurologic signs in the legs.Ann Intern Med. 1968; 69,2:305-8.
7. De Lange EE, Black WC, Mills SE. Radiologic features of retroperitoneal cystic hamartoma.Gastrointest Radiol. 1988; 13,3:266-70.

8. Javery OM, Lee EY. Retroperitoneal hamartoma in a pediatric patient.Pediatr Radiol. 2010; 40,1:15.

9. Eng. C. PTEN: One gene, many syndromes. Hum. Mutat.2003; 22:183-98.

10. Tan WH, Baris HN, Burrows PE ,RobsonCD,Alomari AI,Mulliken JB,et al.The spectrum of vascular anomalies in patients with PTEN mutations: implications for diagnosis and management. J Med Genet .2007;44:594-602 